\title{
Non-equilibration of topological charge and its effects
}

\section{Claude Bernard}

Physics Department, Washington University, Saint Louis, MO 63130, USA

E-mail: cbel lump.wust l . edu

\section{Doug Toussaint*}

Physics Department, University of Arizona, Tucson, AZ 85721, USA

E-mail: doug@physics.arizona.edu

In QCD simulations at small lattice spacings, the topological charge $Q$ evolves very slowly and, if this quantity is not properly equilibrated, we could get incorrect results for physical quantities, or incorrect estimates of their errors. We use the known relation between the dependence of masses and decay constants on the QCD vacuum angle $\theta$ and the squared topological charge $Q^{2}$ together with chiral perturbation theory results for the dependence of masses and decay constants on $\theta$ to estimate the size of these effects and suggest strategies for dealing with them. For the partially quenched case, we sketch an alternative derivation of the known $\chi$ PT results of Aoki and Fukaya, using the nonperturbatively correct chiral theory worked out by Golterman, Sharpe and Singleton, and by Sharpe and Shoresh. With the MILC collaboration's ensembles of lattices with four flavors of HISQ dynamical quarks, we measure the $Q^{2}$ dependence of masses and decay constants and compare to the $\chi \mathrm{PT}$ forms. The observed agreement gives us confidence that we can reliably estimate the errors from slow topology change, and even correct for its leading effects.

34th annual International Symposium on Lattice Field Theory

24-30 July 2016

University of Southampton, UK

\footnotetext{
*Speaker.
} 


\section{Evolution of $Q$}

QCD simulations using (approximately) continuous evolution algorithms show very slow evolution of the topological charge $Q$ when the lattice spacing is small. This is expected, since in the continuum theory $Q$ cannot change in a continuous evolution of the fields. This is a concern for QCD simulations since the distribution of $Q$ may not be properly sampled in a simulation of practical length. Here we study the evolution of $Q$ in the MILC collaboration's ensembles of lattices with a one-loop Symanzik and tadpole improved gauge action and four flavors of highly improved staggered quarks (HISQ). We use chiral perturbation theory to compute the effects of poor sampling of $Q$ on pseudoscalar masses and decay constants, and compare these results to our simulations.

The ensembles we study have lattice spacings ranging from $0.09 \mathrm{fm}$ to $0.03 \mathrm{fm}$, and light sea quark masses either at one fifth of the strange quark mass or approximately tuned to the physical light quark mass. Figure 1 shows the time histories of $Q / V^{1 / 2}$, where $V$ is the four dimensional lattice volume in $\mathrm{fm}^{4}$. In this plot the blue traces are for ensembles with light sea quark mass one fifth of the strange quark mass and red traces for ensembles with physical light quark mass. The increasing autocorrelation time of $Q$ as $a$ gets small is clearly visible, and we see that at $a=0.03$ $\mathrm{fm}$ the simulation has not yet explored most of the desired values of $Q^{2}$. We also see that for each lattice spacing the local structure of the time histories is very similar for the $m_{l}=m_{s} / 5$ ensemble and the physical $m_{l}$ ensemble. However, in the $m_{l}=m_{s} / 5$ ensemble $Q$ ranges over larger values, therefore taking longer to random walk through this range, leading to a longer autocorrelation time. This is as expected, since the gauge action controls the tunneling rate for $Q$, so the average squared change in $Q$ per unit volume per unit simulation time is approximately independent of the light quark mass. However, the fermion determinant does suppress the average $Q^{2}$, and we expect the topological susceptibility, $\left\langle Q^{2} / V\right\rangle$, to be approximately proportional to $m_{l}$.

Figure 2 shows the tunneling rate, $\left\langle(\Delta Q)^{2} /(V d t)\right\rangle$ with octagons, where the blue symbols are for the $m_{s} / 5$ ensembles and the red for the physical $m_{l}$ ensembles. We see that the tunneling rate doesn't depend much on the quark mass, but is decreasing as expected as $a$ gets small. (In the cases where there are two blue octagons, there were two sub-ensembles with a different molecular dynamics trajectory length in each sub-ensemble.) The crosses in Fig. 2 show the topological susceptibility, $\left\langle Q^{2} / V\right\rangle$. Here we see the expected strong dependence on light quark mass. The small error bar on the $0.03 \mathrm{fm}$ point is unrealistic — it simply reflects the fact the $Q$ is basically stuck near this value in this simulation.

\section{Theoretical treatment of the dependence on topological charge}

The topological susceptibility is defined by [1]

$$
\begin{aligned}
Z(\theta) & =\int \mathscr{D} A \mathscr{D} \bar{\Psi} \mathscr{D} \Psi \exp (-S[A, \bar{\Psi}, \Psi]) \exp (-i \theta Q[A]) \\
\chi_{t} & \equiv-\left.\frac{1}{V}\left(\frac{1}{Z} \frac{\partial^{2} Z}{\partial \theta^{2}}\right)\right|_{\theta=0}=\frac{1}{V}\left\langle Q^{2}\right\rangle .
\end{aligned}
$$




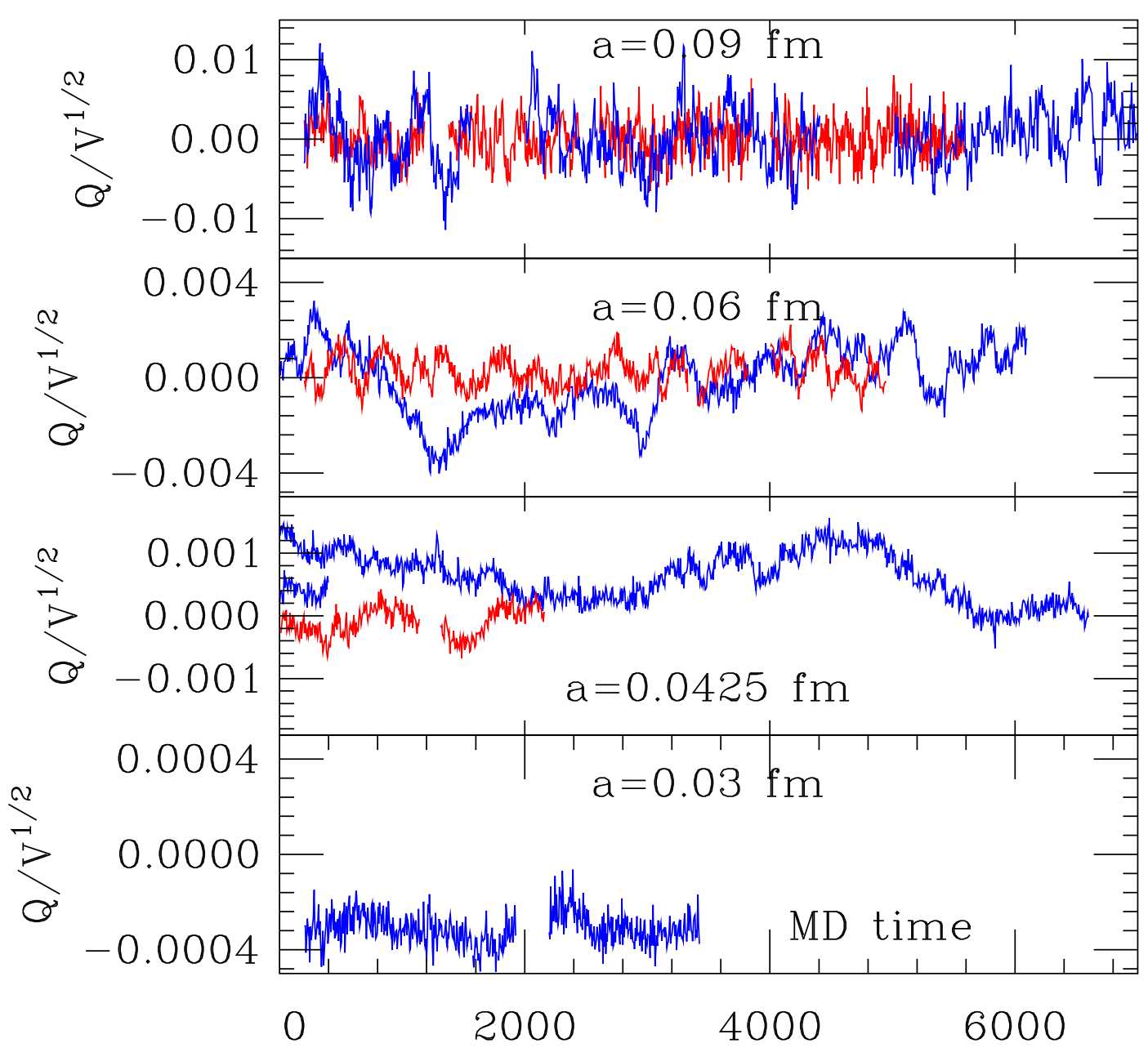

Figure 1: Topological charge time histories for various lattice spacings. Blue traces are for ensembles with light sea quark mass one fifth of the strange quark mass and red traces for ensembles with light sea quark mass at its physical value. Notice the narrower distributions and shorter autocorrelation times for physical quark mass ensembles. Multiple traces in some graphs correspond to multiple runs, sometimes with differing trajectory lengths.

Quantities evaluated at fixed $Q$ are found by Fourier transforming

$$
\begin{aligned}
Z_{Q} & =\frac{1}{2 \pi} \int_{-\pi}^{\pi} d \theta \exp (i \theta Q) Z(\theta) \\
G_{Q} & =\left\langle\mathscr{O}_{1} \mathscr{O}_{2} \ldots \mathscr{O}_{n}\right\rangle_{Q}=\frac{1}{Z_{Q}} \frac{1}{2 \pi} \int_{-\pi}^{\pi} d \theta \exp (i \theta Q) Z(\theta) G(\theta)
\end{aligned}
$$

with $G(\theta)=\left\langle\mathscr{O}_{1} \mathscr{O}_{2} \ldots \mathscr{O}_{n}\right\rangle_{\theta}$. For large 4-dim volumes $V$, we can do the $\theta$ integrals by the saddle point method to find (for $B$ the mass $M$ or the decay constant $f$ ) [2,3]

$$
\left.B\right|_{Q, V}=B+\frac{1}{2 \chi_{T} V} B^{\prime \prime}\left(1-\frac{Q^{2}}{\chi_{T} V}\right)+\mathscr{O}\left(\frac{1}{\left(\chi_{T} V\right)^{2}}\right)
$$

where $\left.B^{\prime \prime} \equiv \frac{\partial^{2} B}{\partial \theta^{2}}\right|_{\theta=0}$. By Eq. (2.2), the correction vanishes when averaged over $Q$.

Since the quantities $M^{\prime \prime}$ and $f^{\prime \prime}$ are physical, we can get a theoretical handle on topological effects by calculating them in continuum, infinite volume, $\chi$ PT. A first calculation of $M^{\prime \prime}$ in $\chi$ PT for 


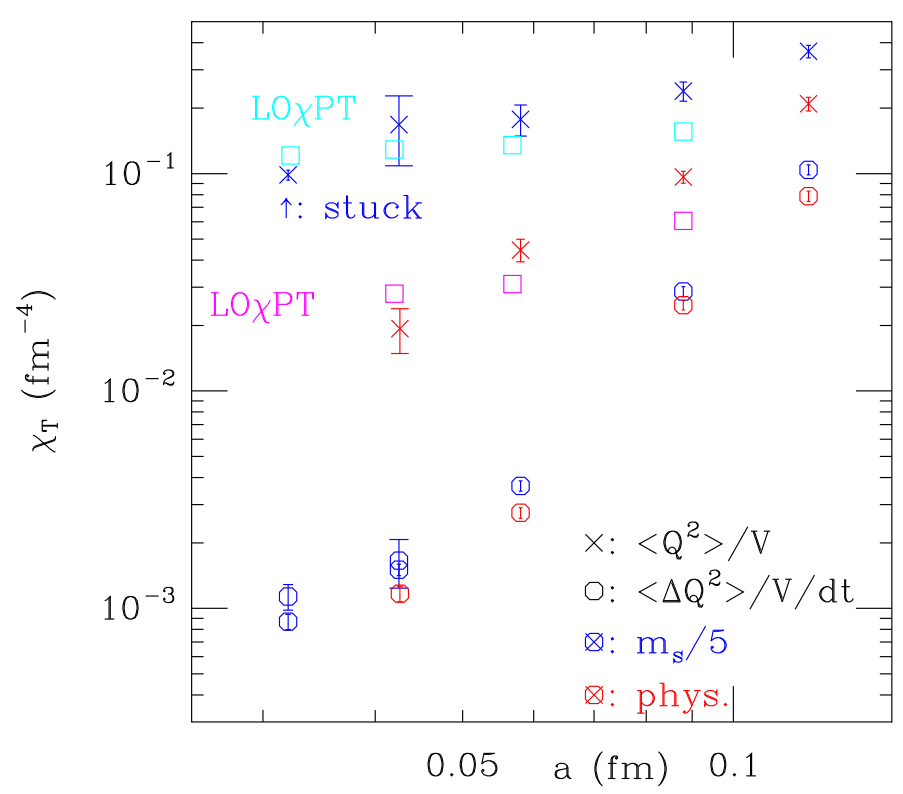

Figure 2: Average topological susceptibility $\left\langle Q^{2} / V\right\rangle$ (crosses) and tunneling rate $\left\langle(\Delta Q)^{2}\right\rangle$ (octagons) versus lattice spacing. The cyan and magenta squares are the lowest order chiral perturbation theory predictions for the susceptibility.

full (unitary) QCD appears in Ref. [2]. Since most of our lattice data is partially quenched, we need to extend the calculation to $M^{\prime \prime}$ and $f^{\prime \prime}$ to partially quenched $\chi \mathrm{PT}$ (PQ $\left.\chi \mathrm{PT}\right)$. Reference [4] worked this out, using the replica method to remove the determinant of the valence quarks. However, the required calculation is non-perturbative, at least on its face, since the vacuum state changes in the presence of $\theta$. The replica method is only justified perturbatively, so a non-perturbatively safe method is preferable. The Lagrangian approach of Ref. [5], which introduces ghost (bosonic) quarks to cancel the valence quark determinant, is also only valid perturbatively, since it ignores the requirement that bosonic path integral be convergent. References [6, 7] fixed the non-perturbative problems of the Lagrangian approach by taking into account the convergence requirement.

The PQ $\chi \mathrm{PT}$ Lagrangian in the presence of $\theta$ for $n_{F}$ sea quarks, $n_{V}$ valence quarks, and $n_{V}$ ghost quarks is $[6,7]$

$$
\mathscr{L}=\frac{f^{2}}{8} \operatorname{str}\left(\partial_{\mu} \Sigma \partial_{\mu} \Sigma^{-1}\right)-\frac{B f^{2}}{4} \operatorname{str}\left(e^{-i \theta / n_{F}} \mathscr{M} \Sigma+e^{i \theta / n_{F}} \mathscr{M} \Sigma^{-1}\right),
$$

where str is the supertrace, the factors $e^{ \pm i \theta / n_{F}}$ arise from an anomalous rotation to remove the $i \theta Q$ term in Eq. (2.1), and $\Sigma$ is an $\left(n_{F}+2 n_{V}\right) \times\left(n_{F}+2 n_{V}\right)$ matrix constructed from the meson field $\Phi$ :

$$
\Sigma=e^{2 i \Phi / f}, \quad \Phi=\left(\begin{array}{cc}
\phi & \bar{\chi} \\
\chi & -i \hat{\phi}
\end{array}\right) .
$$

Here $\phi$ and $\hat{\phi}$ are hermitian bosonic fields ${ }^{1}$, representing quark-quark and ghost-ghost mesons, respectively. The quark-ghost fields $\chi$ and $\bar{\chi}$ are fermionic. The field $\hat{\phi}$ is integrated from $-\infty$ to $+\infty$; the factor $i$ in Eq. (2.5) ensures a convergent path integral, making the $\hat{\phi}$ action positive definite. Convergence for the $\phi$ integral is not a problem because the domain of $\phi$ is a compact space, as usual. Subtleties for fields along the diagonal have been ignored in Eq. (2.5) for simplicity.

\footnotetext{
${ }^{1}$ Technically, this applies to the "body" of $\hat{\phi}$.
} 
In the full theory, we would now minimize the potential energy term to find the vacuum state $\langle\Sigma\rangle$. Here, the potential energy is complex. Reference [6] argues that we should therefore find a saddle point (deforming the $\hat{\phi}$ contour as needed), not a minimum. We note that, unlike what happens in the quenched case [6], the symmetry between valence and ghost quarks is automatically preserved by the saddle point, and does not need to be imposed by hand. To find the $\theta$-dependence of the mass (at tree level), we can then expand the Lagrangian to quadratic order in $\Phi$ around the vacuum state by writing

$$
\Sigma=\sqrt{\langle\Sigma\rangle} e^{2 i \Phi / f} \sqrt{\langle\Sigma\rangle} .
$$

This way of expanding keeps "extended parity" symmetry (parity $+\theta \rightarrow-\theta$ ) simple: $\Phi \rightarrow-\Phi$, $\Sigma \rightarrow \Sigma^{-1}$. For the decay constant, we similarly expand the axial current to linear order in $\Phi$.

For $n_{F}=3$ with masses $m_{u}=m_{d}=m$ and $m_{s}$, and $n_{V}=2$ with masses $m_{x}, m_{y}$, we find

$$
\begin{aligned}
M_{x y}^{\prime \prime} & =-M_{x y} \frac{m^{2} m_{s}^{2}}{2\left(m+2 m_{s}\right)^{2}} \frac{1}{m_{x} m_{y}}, \\
f_{x y}^{\prime \prime} & =-f_{x y} \frac{m^{2} m_{s}^{2}}{4\left(m+2 m_{s}\right)^{2}} \frac{\left(m_{x}-m_{y}\right)^{2}}{m_{x}^{2} m_{y}^{2}} .
\end{aligned}
$$

These results agree with those in Ref. [4]. This indicates that it is not necessary to use a nonperturbatively correct approach for this problem; the reason seems to be that in the end we only need the new vacuum state in the infinitesimal neighborhood of $\theta=0$. The singular limit as $m_{x}$ or $m_{y} \rightarrow 0$ presumably comes from topological zero modes, which are not suppressed by low valence-quark mass since the valence determinant is absent. For $n_{F}=4$, decoupling works (if $m_{c}$ is sufficiently heavy), so we can still use the above results.

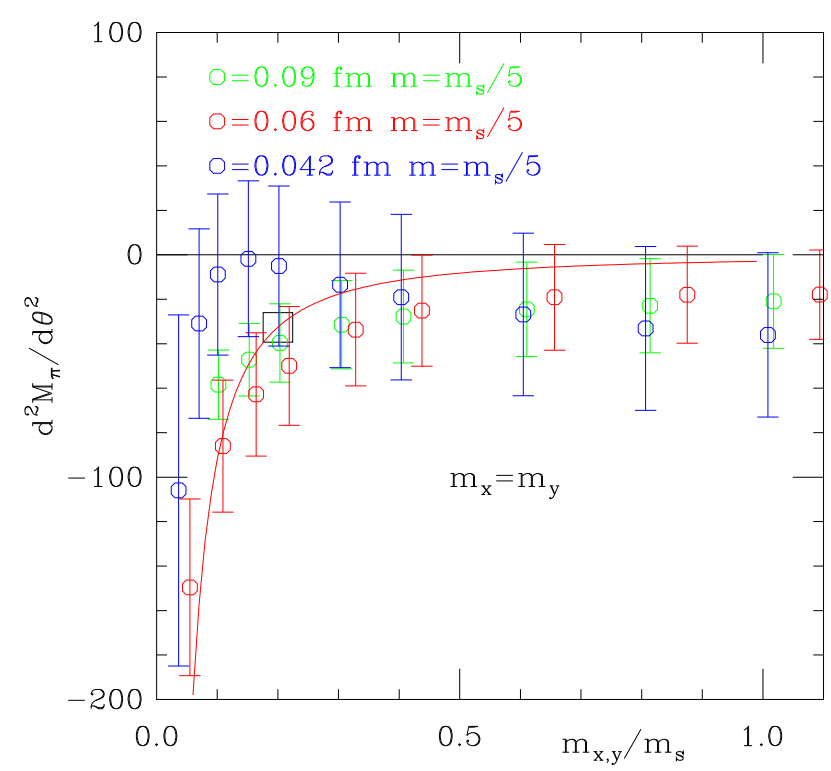

Figure 3: $\frac{\partial^{2} M}{\partial \theta^{2}}$ on ensembles with $m_{l}=m_{s} / 5$ along the line $m_{x}=m_{y}$. The red line is the PQ $\chi$ PT prediction (no free parameters), and the square is the unitary point.

\section{Comparison to simulation results}

We have calculated pseudoscalar masses and decay constants on the HISQ ensembles described above, using methods described in Ref. [8]. To find the dependence on the topological 
charge, we use the results of a single-elimination jackknife analysis of these quantities together with the time histories of topological charge shown above. We first construct effective masses and decay constants for each lattice by taking the ensemble average values minus $N$ times the deviation of the corresponding jackknife sample value from the ensemble average. Then we fit to a linear function of the topological charge, $M=M_{0}+\frac{C}{2} Q^{2}$, assigning each data point an error equal to the standard deviation of the distribution. (Strictly speaking, it should be the standard deviation reduced by the contribution of the dependence on topological charge to the variance, but this turns out to make little difference.) Then we use Eq. 2.3 to convert $C$ into a derivative with respect to $\theta$.

The results are noisy, but consistent with the $\chi P T$ predictions. Statistically significant signals are found in the $m_{l}=m_{s} / 5$ ensembles, since these have much smaller physical volumes than the physical light quark mass ensembles. For example, in the $a \approx 0.06 \mathrm{fm}$ ensembles the $m_{l}=m_{s} / 5$ lattices have a volume of $180 \mathrm{fm}^{4}$, while the physical $m_{l}$ lattices have a volume of $1920 \mathrm{fm}^{4}$. Also, Eq. 2.7 shows that the derivatives of the masses and decay constants have a partially quenched divergence when $m_{x}$ or $m_{y}$ goes to zero with $m_{l}$ fixed, and for the $m_{l}=m_{s} / 5$ ensembles we have used valence quark masses smaller than $m_{l}$, in some cases as small as the physical $m_{l}$.

Figure 3 shows $\frac{\partial^{2} M}{\partial \theta^{2}}$ for the $m_{s} / 5$ ensembles for degenerate valence quark masses, $m_{x}=m_{y}$. The red line in the figure is the $P Q \chi P T$ prediction in Eq. 2.7, which we emphasize is a prediction with no free parameters. Obviously the statistical errors are large, but they are consistent with the prediction, and the divergence at small valence quark mass is clearly seen. Since $\frac{\partial^{2} F}{\partial \theta^{2}}$ vanishes for degenerate valence quarks, we plot this quantity along different lines in Fig. 4. The left panel shows $\frac{\partial^{2} F}{\partial \theta^{2}}$ as a function of one valence quark mass, $m_{x}$, with the other fixed at the strange quark mass, together with the $\chi P T$ prediction. The right panel shows $\frac{\partial^{2} F}{\partial \theta^{2}}$ along lines where $m_{y}$ is held fixed at the lightest valence quark mass available in each ensemble. The vanishing of $\frac{\partial^{2} F}{\partial \theta^{2}}$ when the valence quarks are degenerate is particularly striking in this plot.

Knowing the dependence of masses and decay constants on the average $Q^{2}$, we can correct our simulation results to account for the difference of the average in our simulation, $\left\langle Q^{2}\right\rangle_{\text {sample }}$ and the correct $\left\langle Q^{2}>\right.$. To estimate this correct $\left\langle Q^{2}\right\rangle$ we use the lowest order $\chi P T$ result, $\chi_{T}=\frac{f_{\pi}^{2}}{4} \overline{M_{I}^{2}}$ where $1 / \overline{M_{I}^{2}}=2 / M_{\pi, I}^{2}+1 / M_{s s, I}^{2}$ [1]. Here the " $I$ " indicates the taste singlet masses [9]. The $\chi P T$ results are shown in Fig. 2. For large $a$ the deviation from the lowest order $\chi P T$ results is due to lattice artifacts, probably mostly higher order taste breaking effects, but for $a=0.042$ and $0.03 \mathrm{fm}$ we expect the $\chi P T$ results to be pretty good. For an example of the size of these effects in our simulation, we look at $f_{K} / f_{\pi}$ in our two ensembles with $a \approx 0.042 \mathrm{fm}$. This ratio has very small statistical errors, so this is a good test. To make this correction, rearrange Eq. 2.3 as

$$
f_{\text {corrected }}=f_{\text {sample }}-\frac{1}{2 \chi_{T} V} F^{\prime \prime}\left(1-\frac{<Q^{2}>_{\text {sample }}}{\chi_{T} V}\right)
$$

For the $0.042 \mathrm{fm}$ physical $m_{l}$ ensemble, with $L=6.05 \mathrm{fm}$ and estimating $\frac{\left\langle Q^{2}>_{\text {sample }}\right.}{\chi_{T} V} \approx 0.7$ we find a fractional shift $\frac{\Delta f}{f}=0.0002$. This can be compared to our statistical error on this ratio, 0.0010 and to the "conventional" finite size effects from pions propagating around the periodic lattice, estimated in NLO staggered $\chi P T$, of 0.0009 . The effects are larger in the ensemble with $m_{l} / m_{s}=0.2$, since these lattices have much smaller volume and a partial quenching divergence. In this case a similar estimate gives $\frac{\Delta f}{f} \approx-0.002$ to be compared with a statistical error of 0.003 . 

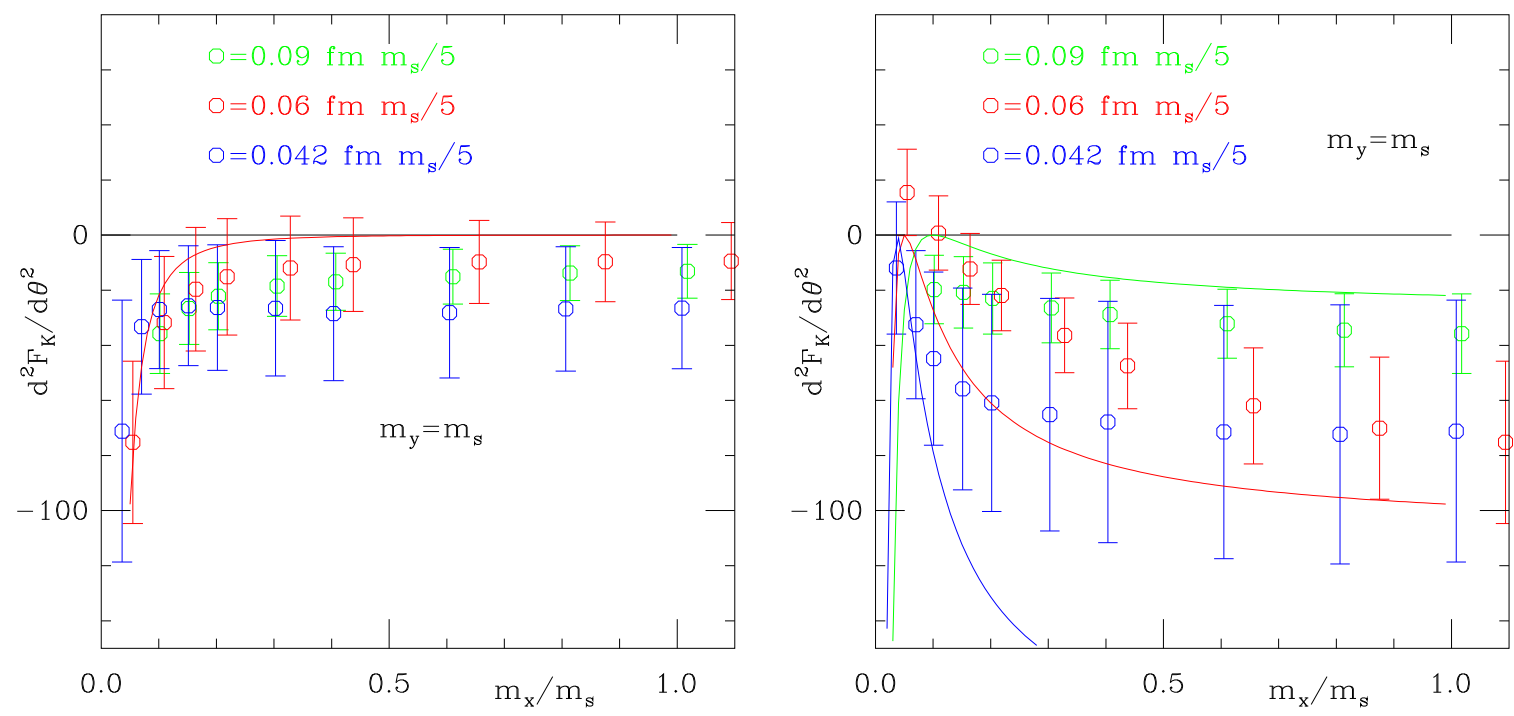

Figure 4: $\frac{\partial^{2} F}{\partial \theta^{2}}$ on ensembles with $m_{l}=m_{s} / 5$. The left panel shows $\frac{\partial^{2} F}{\partial \theta^{2}}$ as a function of one valence quark mass, $m_{x}$ along the line $m_{y}=m_{s}$. The red line is the PQ $\chi \mathrm{PT}$ prediction (no free parameters), which vanishes for degenerate quarks. The right panel shows the quantity with $m_{y}$ fixed to the smallest available value. The lines are the PQ $\chi \mathrm{PT}$ predictions. There are three separate lines in the right panel because the smallest valence quark mass was different in each ensemble, $0.1 m_{s}, 0.05 m_{s}$ and $0.037 m_{s}$ for the $0.09,0.06$ and 0.042 fm ensembles respectively.

We close by noting that this strategy is in the same spirit as our treatment of "conventional" finite size effects. We use $\chi P T$ to estimate the effects and correct our results, and estimates of the effects of higher order $\chi P T$ and/or uncertainties in the $\chi P T$ parameters should be included in the systematic error budget.

\section{Acknowledgements}

This work was supported by US DOE contracts DE-FG02-91ER40628 and DE-FG02-13ER41976. Computations were done at centers supported by the US DOE and NSF, including ALCF, NCSA Bluewaters, NERSC, TACC, NCAR and USQCD facilities.

\section{References}

[1] H. Leutwyler and A.V. Smilga, Phys. Rev. D 46, 5607 (1992).

[2] R. Brower, S. Chandrasekharan, J.W. Negele and U.-J. Wiese, Phys. Lett. B. 560, 64 (2003) [hep-lat/0302005].

[3] S. Aoki, H. Fukaya, S. Hashimoto and T. Onogi, Phys. Rev. D76, 054508 (2007 [arXiv:0707.0396].

[4] S. Aoki and H. Fukaya, Phys. Rev. D 81, 034022 (2010).

[5] C. W. Bernard and M. F. L. Golterman, Phys. Rev. D 49, 486 (1994) [hep-lat/9306005].

[6] M. Golterman, S. Sharpe and R. Singleton, Jr., Phys. Rev. D 71, 094503 (2005) [hep-lat/0501015].

[7] S. Sharpe and N. Shoresh, Phys. Rev. D 64, 114510 (2001) [hep-lat/0108003].

[8] A. Bazavov et al., Phys. Rev. D 90, 074509 (2014), [arXiv:1407.3772].

[9] C. Aubin and C. Bernard, Phys. Rev. D 68, 034014 (2003); B. Billeter, C.E. Detar and J. Osborn, Phys. Rev. D 70, 077502 (2004). 